\title{
Theory of polarization and spatial information recovery by modal dispersal and phase conjugation
}

\author{
Yasuo Tomita, Ram Yahalom, and Amnon Yariv \\ Department of Applied Physics, 128-95, California Institute of Technology, Pasadena, California 91125
}

Received July 14, 1987; accepted November 16, 1987

\begin{abstract}
A general theory of polarization and spatial information recovery by modal dispersal and phase conjugation is presented by means of a coherency matrix formalism. The theory is applied to a system that consists of a multimode modal-scrambling fiber terminated by a conventional phase-conjugate mirror that reflects only one polarization component. The degree of polarization and the signal-to-noise ratio of the reconstructed field are discussed as a function of input-beam launching conditions. Some experimental results are also shown for comparison with the theory.
\end{abstract}

\section{INTRODUCTION}

It is well known that when a polarized laser beam is launched into a multimode modal-scrambling fiber, the initially coupled fiber modes are scrambled among all the fiber modes, including those of orthogonal polarization by strong intermodal coupling, and consequently the output beam from the fiber shows speckled spatial structures and depolarization. ${ }^{1}$ These types of beam aberration, i.e., wave-front distortion and polarization scrambling, can be corrected if the aberrated fields including both orthogonal polarizations are phase conjugated. ${ }^{2-8}$ However, it was found recently that even when only one polarization component of the forward-traveling beam from the fiber was reflected by an ordinary phase-conjugate mirror (PCM) and fed back into the fiber, the resultant beam, emerging from the input plane of the fiber, could be a phase-conjugate replica of the original input beam including its original polarization state, i.e., this would be true phase conjugation of vector wave fronts. ${ }^{9}$

Since this first observation in such a fiber-coupled phaseconjugate mirror (FCPCM), a theoretical mode ${ }^{10}$ was proposed, and a number of new applications, including correction of nonreciprocal distortions, ${ }^{11}$ correction of lossy amplitude distortions, ${ }^{12}$ temporal data channeling between beams, ${ }^{13}$ and all-optical beam thresholding, ${ }^{14}$ have been reported. In addition, the fidelity of the true phase conjugation using the FCPCM was also studied, ${ }^{15}$ and it was found that the fidelity was strongly dependent on launching conditions of an input beam [i.e., input-beam numerical apertures (N.A.'s)]: if an input-beam N.A. is close to the fiber's N.A., then the reflected beam within such an input-beam N.A. carries nearly the same amount of the noise power as that of the true phase-conjugate beam, resulting in the degradation of polarization and spatial information recovery. Although this input-beam N.A. dependence was explained by a phenomenological model ${ }^{15}$ and its asymptotic case (i.e., a case of a large N.A. input) was also reported, ${ }^{16}$ a detailed analysis has not been given so far.

In this paper we present a general theoretical description of polarization and spatial information recovery using the
FCPCM by means of a coherency matrix formalism. The treatment involves the analysis of the polarization state of the field transmitted through the fiber and the dependence of the polarization recovery and the signal-to-noise ratio (SNR) of the phase-conjugate field on the input-beam N.A. The effects of the modal-scrambling property of the fiber and the fidelity of phase conjugation by the PCM on the polarization recovery are also discussed. The physical processes considered in the present analysis are twofold: (1) a (time-reversed) phase-conjugation process, which is deterministic in nature; and (2) a scattering process in the fiber, which results from partial phase conjugation of the modescrambled field and is seemingly completely random (or stochastic) but is in fact constrained by the unitarity condition of the scattering matrix (i.e., the energy-conservation condition). In this case we take the coupling strength in this scattering process to be essentially the same among all the fiber guided modes but its relative phases to be random under the constraint of the unitarity condition. In the analysis of the polarization properties of the phase-conjugate field, unlike in the treatment of the Jones calculus for random media, ${ }^{17}$ we do not resort to a statistical ensemble average over the coherency matrix elements but use the modal averaging ${ }^{10}$ over phase-mismatched fields in this phase-conjugation process, which may be analogous to the phase-matching condition in the coupled-mode theory. ${ }^{18}$ The main reason for our approach is that one usually treats only a single fiber in phase-conjugation experiments. In the SNR treatment, however, we simplify the analysis by using an a priori knowledge of the statistical properties of a (speckle) noise field instead of considering statistical properties of the scattering matrix and its relation to the properties of the noise field. This is done by assuming a probability-density function of the outcoupled noise field in the free space, and the SNR can then be obtained from the rootmean-square (rms) value of (statistical) intensity fluctuations of the noise field.

With the above treatment the present analysis enables us to evaluate theoretically the fidelity of polarization and spatial information recovery by using the FCPCM and to give a 
criterion for the limitation of the use of the FCPCM. Some experimental results are also shown for comparison with the theory.

\section{BASIC FORMULATION USING SCATTERING MATRICES}

Figure 1 shows a schematic of the FCPCM. An imagebearing incident field $\mathbf{E}^{(1)}$ is launched into a multimode modal-scrambling fiber, which is assumed to be linear with negligible loss. Because of the strong intermodal coupling in the fiber, the input power initially coupled into any one fiber guided mode is distributed essentially uniformly among all the other spatial and polarization modes during propagation, and the outcoupled beam $\mathbf{E}^{(2)}$ from the fiber exhibits speckled spatial structures and nearly complete depolarization. The PCM, e.g., a self-pumped PCM, ${ }^{19}$ is placed after a polarizer (set to the $x$ direction) and phase conjugates only the $x$ component of the field $\mathbf{E}^{(2)}$. The phase-conjugate field $\mathbf{E}^{(3)}$ retraces the original path and is launched into the output side of the fiber. After the propagation and the strong intermodal coupling in the fiber, the left-traveling field forms the output field $\mathbf{E}^{(4)}$ at the input end of the fiber.

By using the same notation as in Ref. 10, the input field $\mathbf{E}^{(1)}$ is expressed, in terms of the fiber guided modes, as

$$
\begin{aligned}
\mathbf{E}^{(1)} & =\sum_{n=1}^{N}\left[a_{x n}^{(1)} \mathbf{e}_{x n}+a_{y n}^{(1)} \mathbf{e}_{y n}\right] \\
& \equiv\left[\begin{array}{c}
A_{x}^{(1)} \\
A_{y}^{(1)}
\end{array}\right],
\end{aligned}
$$

where $N$ is the total number of the fiber guided modes in one polarization; $\mathbf{e}_{x n}$ is the $n$th transverse fiber guided mode, which is predominantly $x$ polarized; $\mathbf{e}_{y n}$ is the $n$th $y$-polarized mode; and $A_{x}^{(1)}$ and $A_{y}^{(1)}$ are column vectors of rank $N$ whose elements are the complex amplitudes $a_{x n}^{(1)}$ and $a_{y n}^{(1)}$, respectively. Note that we neglect the coupling into other possible fiber modes (e.g., leaky and radiation modes) for simplicity of the analysis.

The output field $\mathbf{E}^{(4)}$ is expressed as

$$
\mathbf{E}^{(4)}=r M^{\prime} C M^{*}\left[\mathbf{E}^{(1)}\right]^{*},
$$

where $r$ is the PCM amplitude reflectivity, $M$ is the scattering matrix of the fiber in the forward direction given by

$$
M=\left[\begin{array}{ll}
M_{x x} & M_{x y} \\
M_{y x} & M_{y y}
\end{array}\right],
$$

in which $M_{i j}(i, j=x, y)$ are $N \times N$ submatrices, and $M^{\prime}$ is the scattering matrix in the backward direction. In addition, the matrix $C$, representing the removal of the $y$ polarization by the polarizer, is given by

$$
C=\left[\begin{array}{ll}
I & 0 \\
0 & 0
\end{array}\right],
$$

where $I$ is an $N \times N$ unit matrix. We note that a modeindependent (scalar) reflectivity of the PCM is assumed in Eq. (2). If a mode-dependent reflectivity is taken into account, $r$ should be replaced by a $2 N \times 2 N$ matrix. We discuss this effect in Section 4. In what follows we examine
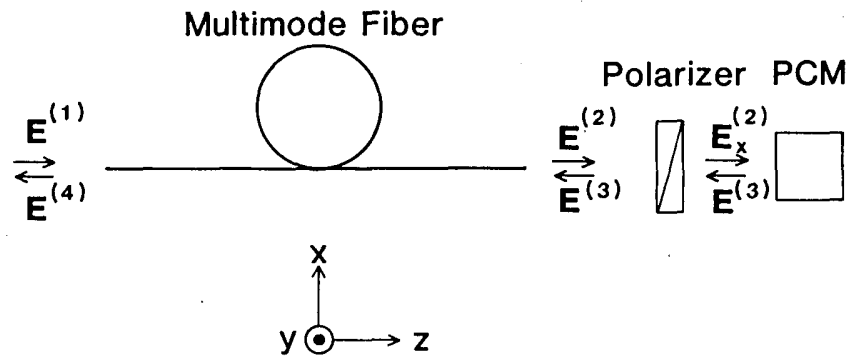

Fig. 1. Schematic of the FCPCM for polarization and spatial information recovery. The (polarization- and modal-scrambling) multimode fiber is assumed to be linear with negligible loss.

the properties of the scattering matrices and express the fields $\mathbf{E}^{(2)}$ and $\mathbf{E}^{(4)}$ in terms of the scattering matrix elements.

Because of the conservation of the energy in a lossless linear fiber, we require the following unitarity condition ${ }^{10}$ :

$$
M^{\dagger} M=\left[\begin{array}{ll}
I & 0 \\
0 & I
\end{array}\right],
$$

where ${ }^{\dagger}$ denotes the Hermite transpose operation. By using Eq. (3), Eq. (5) can be translated into the following sum rules:

$$
\begin{aligned}
& \left(M_{x x}\right)_{i k}\left(M_{x x}\right)_{i k^{\prime}}^{*}+\left(M_{y x}\right)_{i k}\left(M_{y x}\right)_{i k^{\prime}}^{*}=\delta_{k k^{\prime}}, \\
& \left(M_{y y}\right)_{i k}\left(M_{y y}\right)_{i k^{\prime}}^{*}+\left(M_{x y}\right)_{i k}\left(M_{x y}\right)_{i k^{\prime}}^{*}=\delta_{k k^{\prime}}, \\
& \left(M_{x y}\right)_{i k}\left(M_{x x}\right)_{i k^{\prime}}^{*}+\left(M_{y y}\right)_{i k}\left(M_{y x}\right)_{i k^{\prime}}^{*}=0, \\
& \left(M_{x x}\right)_{i k}\left(M_{x y}\right)_{i k^{\prime}}^{*}+\left(M_{y x}\right)_{i k}\left(M_{y y}\right)_{i k^{\prime}}^{*}=0,
\end{aligned}
$$

and

$$
\begin{aligned}
& \left(M_{x x}\right)_{k i}\left(M_{x x}\right)_{k^{\prime} i}^{*}+\left(M_{x y}\right)_{k i}\left(M_{x y}\right)_{k^{\prime} i}^{*}=\delta_{k k^{\prime}}, \\
& \left(M_{y y}\right)_{k i}\left(M_{y y}\right)_{k^{\prime} i}^{*}+\left(M_{y x}\right)_{k i}\left(M_{y x}\right)_{k^{\prime} i}^{*}=\delta_{k k^{\prime}}, \\
& \left(M_{y x}\right)_{k i}\left(M_{x x}\right)_{k^{\prime} i}^{*}+\left(M_{y y}\right)_{k i}\left(M_{x y}\right)_{k^{\prime} i}^{*}=0, \\
& \left(M_{x x}\right)_{k i}\left(M_{y x}\right)_{k^{\prime} i}^{*}+\left(M_{x y}\right)_{k i}\left(M_{y y}\right)_{k^{\prime} i}^{*}=0,
\end{aligned}
$$

where henceforth summation over repeated indices is understood.

For the time-reversal symmetry of any fields in a lossless linear fiber we also require that ${ }^{9,10}$

$$
M^{\prime} M^{*}=\left[\begin{array}{ll}
I & 0 \\
0 & I
\end{array}\right] .
$$

From Eqs. (5) and (8) we obtain

$$
M^{\prime}=M^{t},
$$

where $t$ denotes the transpose operation. By using the submatrices given in Eq. (3), Eq. (9) can be rewritten as

$$
\begin{aligned}
\left(M_{x x}^{\prime}\right)_{i j} & =\left(M_{x x}\right)_{j i}, \\
\left(M_{y y}^{\prime}\right)_{i j} & =\left(M_{y y}\right)_{j i}, \\
\left(M_{x y}^{\prime}\right)_{i j} & =\left(M_{y x}\right)_{j i}, \\
\left(M_{y x}^{\prime}\right)_{i j} & =\left(M_{x y}\right)_{j i} .
\end{aligned}
$$

Here we note that the elements of the scattering matrices are interrelated by the constraint given by Eqs. (6), (7), and (10). 


\section{SPATIAL AND POLARIZATION PROPERTIES OF THE FIELD $E^{(2)}$}

With the relation $\mathbf{E}^{(2)}=M \mathbf{E}^{(1)}$, we express the correlations between the $2 N$ modes of the field $\mathrm{E}^{(2)}$ by means of the following $2 N \times 2 N$ Hermitian coherency matrix:

$$
\begin{aligned}
L^{(2)} & \equiv\left\langle\mathbf{E}^{(2)} \mathbf{E}^{(2)^{\dagger}}\right\rangle \\
& =M\left\langle\mathbf{E}^{(1)} \mathbf{E}^{(1)^{\dagger}}\right\rangle M^{\dagger}=\left[\begin{array}{ll}
L_{x x}^{(2)} & L_{x y}^{(2)} \\
L_{x y}^{(2)^{\dagger}} & L_{y y}^{(2)}
\end{array}\right],
\end{aligned}
$$

where $\langle\ldots\rangle$ denotes the time average and $L_{i j}^{(2)}(i, j=x, y)$ are $N \times N$ matrices given by

$$
\begin{aligned}
L_{x x}^{(2)}= & M_{x x} L_{x x}^{(1)} M_{x x}^{\dagger}+M_{x x} L_{x y}^{(1)} M_{x y}^{\dagger} \\
& +M_{x y} L_{x y}^{(1)^{\dagger}} M_{x x}^{\dagger}+M_{x y} L_{y y}^{(1)} M_{x y}^{\dagger}, \\
L_{y y}^{(2)}= & M_{y x} L_{x x}^{(1)} M_{y x}^{\dagger}+M_{y x} L_{x y}^{(1)} M_{y y}^{\dagger} \\
& +M_{y y} L_{x y}^{(1)} M_{y x}^{\dagger}+M_{y y} L_{y y}^{(1)} M_{y y}^{\dagger}, \\
L_{x y}^{(2)}= & M_{x x} L_{x x}^{(1)} M_{y x}^{\dagger}+M_{x x} L_{x y}^{(1)} M_{y y}^{\dagger} \\
& +M_{x y} L_{x y}^{(1)^{\dagger}} M_{y x}^{\dagger}+M_{x y} L_{y y}^{(1)} M_{y y}^{\dagger},
\end{aligned}
$$

in which $L^{(1)} \equiv\left\langle\mathbf{E}^{(1)} \mathbf{E}^{(1)^{\dagger}}\right\rangle$ denotes the correlations between the $2 N$ modes of the input field $\mathbf{E}^{(1)}$. We note that the effect of a possible decrease of the temporal coherence of the light source at the output, which is due to the modal dispersion in the fiber, ${ }^{20}$ is not taken into account in the present analysis.

We now introduce the following modified $2 \times 2$ coherency matrix:

$$
J^{(2)} \equiv\left[\begin{array}{ll}
J_{x x}^{(2)} & J_{x y}^{(2)} \\
J_{x y}^{(2)^{*}} & J_{y y}^{(2)}
\end{array}\right] .
$$

Here each element $J_{i j}^{(2)}(i, j=x, y)$ is given by

$$
\begin{aligned}
J_{i j}^{(2)} & =\sum_{k=1}^{N} \sum_{l=1}^{N} \iint_{\sigma}\left[L_{i j}^{(2)}\right]_{k l} e_{i k} e^{*}{ }_{j l} \mathrm{~d} x \mathrm{~d} y \\
& =\text { (const.) } \times \sum_{k=1}^{N}\left[L_{i j}^{(2)}\right]_{k k} \\
& =\text { (const.) } \times \operatorname{Tr}\left[L_{i j}^{(2)}\right] .
\end{aligned}
$$

In Eq. (13b) Tr denotes a trace of a matrix, and we have used the orthogonality of the fiber modes, ${ }^{21}$ i.e., $\iint_{\sigma} e_{i m} e^{*}{ }_{j n} \mathrm{~d} x \mathrm{~d} y=$ (constant) $\times \delta_{m n}(i, j=x, y ; m, n=1, \ldots, N)$, where $\sigma$ denotes the whole fiber cross section and a circular fiber is assumed, so that we can neglect the contributions of the offdiagonal elements of $L_{x x}^{(2)}, L_{x y}^{(2)}$, and $L_{y y}^{(2)}$ to $J^{(2)}$ on the detection of the field $\mathbf{E}^{(2)}$ over $\sigma$. We note that, unlike in the usual definition of the coherency matrix, ${ }^{22}$ the elements of $J^{(2)}$ have the dimensionality of power [hereafter, however, we shall omit the constant in Eq. (13b) for brevity].

For the sake of simplicity we consider the $x$-polarized input here. Then, with $L_{x y}^{(1)}=L_{y y}^{(1)}=0$, we can write the diagonal elements in Eqs. (12) as follows:

$$
\begin{aligned}
{\left[L_{x x}^{(2)}\right]_{i i} } & =\left(M_{x x}\right)_{i k}\left(M_{x x}\right)_{i k^{\prime}}^{*}\left[L_{x x}^{(1)}\right]_{k k^{\prime}} \\
& =\left|\left(M_{x x}\right)_{i k}\right|^{2}\left[L_{x x}^{(1)}\right]_{k k}+\left(M_{x x}\right)_{i k}\left(M_{x x}\right)_{i k^{\prime}}^{*}\left[L_{x x}^{(1)}\right]_{\substack{k k^{\prime} \\
\left(k \neq k^{\prime}\right)}},
\end{aligned}
$$

$$
\left[L_{y y}^{(2)}\right]_{i i}=\left|\left(M_{y x}\right)_{i k}\right|^{2}\left[L_{x x}^{(1)}\right]_{k k}+\left(M_{y x}\right)_{i k}\left(M_{y x}\right)_{i k^{\prime}}^{*}\left[L_{x x}^{(1)}\right]_{k k^{\prime}},
$$

$\left[L_{x y}^{(2)}\right]_{i i}=\left(M_{x x}\right)_{i k}\left(M_{y x}\right)_{i k^{\prime}}^{*}\left[L_{x x}^{(1)}\right]_{k k^{\prime}}$

As was mentioned in Section 1, we assume that, because of the strong intermodal coupling in the fiber, the amplitudes of the matrix elements $M_{i j}$, i.e., the coupling strength between modes, are essentially the same (or symmetrically and widely distributed with respect to the diagonal elements $M_{i i}$, while their relative phases are distributed essentially uniformly over the $-\pi-+\pi$ interval (henceforth we refer to this as the random coupling approximation; see Appendix A). Then we see from Eqs. (14) that the input power initially coupled into any one fiber guided mode is redistributed among all the other fiber guided modes, including those of the orthogonal $y$ polarization during propagation. In addition, the out-coupled different spatial modes possessing random phases interfere with one another at any point, resulting in the speckled spatial structures in the free space.

The polarization state of the field $\mathbf{E}^{(2)}$ can be obtained by using $J^{(2)}$. From Eqs. (13) and (14) we have

$$
\begin{aligned}
& J_{x x}^{(2)}=a_{k k}\left[L_{x x}^{(1)}\right]_{k k}+a_{k k^{\prime}}\left[L_{x x}^{(1)}\right]_{\substack{k k^{\prime} \\
\left(k \neq k^{\prime}\right)}}, \\
& J_{y y}^{(2)}=\left(\delta_{k k}-a_{k k}\right)\left[L_{x x}^{(1)}\right]_{k k}-a_{k k^{\prime}}\left[L_{x x}^{(1)}\right]_{\substack{k k^{\prime} \\
\left(k \neq k^{\prime}\right)}}, \\
& J_{x y}^{(2)}=b_{k k^{\prime}}\left[L_{x x}^{(1)}\right]_{k k^{\prime}},
\end{aligned}
$$

where we used the sum rules given in Eqs. (6) and introduced the following parameters:

$$
a_{k k^{\prime}} \equiv\left(M_{x x}\right)_{i k}\left(M_{x x}\right)_{i k^{\prime}}^{*}
$$

and

$$
b_{k k^{\prime}} \equiv\left(M_{x x}\right)_{i k}\left(M_{y x}\right)_{i k^{\prime}}^{*}
$$

Here we note that the terms $a_{\substack{k k^{\prime} \\\left(k \neq k^{\prime}\right)}}$ and $b_{k k^{\prime}}$ in Eqs. (16) are much smaller than $a_{k k}$ because of the modal averaging in the random-coupling approximation. The Stokes parameters $\left(s_{0}, s_{1}, s_{2}, s_{3}\right)$ and the degree of polarization $\left[P^{(2)}\right]$ (Ref. 22) of the field $\mathbf{E}^{(2)}$ are then given by

$$
\begin{aligned}
& s_{0} \equiv J_{x x}^{(2)}+J_{y y}^{(2)}=\sum_{k=1}^{N}\left[L_{x x}^{(1)}\right]_{k k} \\
& s_{1} \equiv J_{x x}^{(2)}-J_{y y}^{(2)}=2 a_{k k}\left[L_{x x}^{(1)}\right]_{k k}+2 a_{k k^{\prime}}\left[L_{x x}^{(1)}\right]_{\substack{k k^{\prime} \\
\left(k \neq k^{\prime}\right)}}-s_{0} \\
& s_{2} \equiv J_{x y}^{(2)}+J_{y x}^{(2)}=2 \operatorname{Re}\left\{b_{k k^{\prime}}\left[L_{x x}^{(1)}\right]_{k k^{\prime}}\right\} \\
& s_{3} \equiv i\left[J_{y x}^{(2)}-J_{x y}^{(2)}\right]=2 \operatorname{Im}\left\{b_{k k^{\prime}}\left[L_{x x}^{(1)}\right]_{k k^{\prime}}\right\}
\end{aligned}
$$

and

$$
P^{(2)}=\frac{\left(s_{1}^{2}+s_{2}^{2}+s_{3}^{2}\right)^{1 / 2}}{s_{0}} .
$$

By using the random-coupling approximation, i.e., $a_{k k} \simeq 0.5$ for any $k, P^{(2)}$ can be reduced to

$$
p^{(2)} \simeq\left(q^{2}+|u|^{2}\right)^{1 / 2}
$$

where 
Table 1. Experimental Data of the Stokes Parameters and the Degree of Polarization of the Field $\mathbf{E}^{(2)^{a}}$

\begin{tabular}{ccccc}
\hline Input-Beam N.A. & $s_{1} / s_{0}$ & $s_{2} / s_{0}$ & $s_{3} / s_{0}$ & $\mathrm{P}^{(2)}$ \\
\hline 0.02 & 0.003 & 0.008 & -0.016 & 0.018 \\
0.11 & 0.033 & 0.023 & -0.028 & 0.049 \\
0.25 & 0.004 & 0.041 & -0.021 & 0.046 \\
\hline
\end{tabular}

${ }^{a}$ In the experiment a multimode (N.A.fiber $=0.29 ; 5 \mathrm{~m}$ long) graded-index fiber and a linearly polarized input $(\lambda=5145 \AA)$ were used.

$$
q \equiv \frac{2 a_{k k^{\prime}}\left[L_{x x}^{(1)}\right]_{\substack{k k^{\prime} \\\left(k \neq k^{\prime}\right)}}}{s_{0}}
$$

and

$$
u \equiv \frac{2 b_{k k^{\prime}}\left[L_{x x}^{(1)}\right]_{k k^{\prime}}}{s_{0}} .
$$

We thus find that $q$ and $u$, which are expressed by $a_{k k^{\prime}}$ and $b_{k k^{\prime}}$, respectively, are responsible for the residual polarization of the field $\mathbf{E}^{(2)}$. Table 1 shows the experimental data of the Stokes parameters and the degree of polarization of the field $\mathbf{E}^{(2)}$ for the different values of input-beam N.A.'s. It is seen that $s_{1}, s_{2}$, and $s_{3}$ are much smaller than $s_{0}$; therefore the degree of polarization $P^{(2)}$ is much smaller than unity, i.e., $|q|$ and $|u|$ are much smaller than unity, and consequently the field $\mathbf{E}^{(2)}$ is almost completely depolarized, independently of the input-beam N.A.'s. These data clarify the validity of the random-coupling approximation and the assumption of the modal averaging by which the cross terms $a_{k k^{\prime}}$ and $b_{k k^{\prime}}$ are much smaller than unity. We note that the parameter $q$ will play an important role in the fidelity of the reconstruction of the original information, as we discuss in Section 4.

\section{SPATIAL AND POLARIZATION PROPERTIES OF THE FIELD $\mathrm{E}^{(4)}$}

In Section 3 we showed that the field $\mathbf{E}^{(2)}$ suffers spatial distortions and nearly complete depolarization because of the strong intermodal coupling in the fiber. In this section we show that such a distorted and depolarized field can be corrected, under certain conditions, even when only one polarization component of the field $\mathbf{E}^{(2)}$ is phase conjugated.

First we rewrite Eq. (2) as

$$
\mathbf{E}^{(4)}=r S\left[\mathbf{E}^{(1)}\right]^{*},
$$

where the scattering matrix $S$ in the round-trip propagation is given by $S=M^{\prime} C M^{*}$. Here we again use the randomcoupling approximation, i.e., $\sum_{i=1}^{N}\left|\left(M_{x x}\right)_{i k}\right|^{2}, \sum_{i=1}^{N}\left|\left(M_{x y}\right)_{i k}\right|^{2} \simeq$ 0.5. Then $S=S_{1}+S_{2}$, where

$$
S_{1}=\frac{1}{2}\left[\begin{array}{ll}
I & 0 \\
0 & I
\end{array}\right]
$$

and

$$
S_{2}=\left[\begin{array}{ll}
D & Q \\
Q^{\dagger} & D^{\prime}
\end{array}\right],
$$

in which $D, D^{\prime}$, and $Q$ are $N \times N$ submatrices given by

$$
\begin{aligned}
D_{i j} & = \begin{cases}0 & (i=j) \\
\left(M_{x x}\right)_{k i}\left(M_{x x}\right)_{k j}^{*} & (i \neq j),\end{cases} \\
D_{i j}^{\prime} & =\left\{\begin{array}{ll}
0 & (i=j) \\
-\left(M_{y y}\right)_{k i}\left(M_{y y}\right)_{k j}^{*} & (i \neq j)
\end{array},\right. \\
Q_{i j} & =\left(M_{x x}\right)_{k i}\left(M_{x y}\right)_{k j}^{*} .
\end{aligned}
$$

Then the field $\mathbf{E}^{(4)}$ given by Eq. (21) becomes

$$
\mathbf{E}^{(4)}=1 / 2 r\left[\mathbf{E}^{(1)}\right]^{*}+\mathbf{V}
$$

where $\mathbf{V}=r S_{2}\left[\mathbf{E}^{(1)}\right]^{*}$. The first term on the right-hand side of Eq. (24) corresponds to the true phase-conjugate replica of the input field $\mathbf{E}^{(1)}$, while the second term corresponds to the noise-possessing random phases in the field $\mathbf{E}^{(4)}$.

Figure 2 shows a diagrammatic explanation of the formation of the field $\mathbf{E}^{(4)}$. The $i$ th fiber guided mode of the $x$ polarization that is excited initially at the input plane of the fiber is coupled into all the fiber guided modes at the output plane in the forward direction. After the elimination of the $y$-polarized component and phase conjugation of the $x$-polarized component, each mode at the output plane is, again, coupled into all the fiber guided modes at the input plane in the backward direction. In Fig. 2(a) the (time-reversed) paths in the backward direction are deterministic and are exactly the same as those in the forward direction, resulting in a constructive coherent superposition of the scattered fields at each mode at the input plane. Because of the constructive interference this true phase-conjugate field, corresponding to the term $1 / 2 r\left[\mathbf{E}^{(1)}\right] *$ in Eq. (24), has almost one half of the total reflected power. [Note the factor $1 / 2$ in Eq. (24) and remember that almost one half of the power of the field $\mathbf{E}^{(2)}$ is eliminated by the polarizer and the remain-
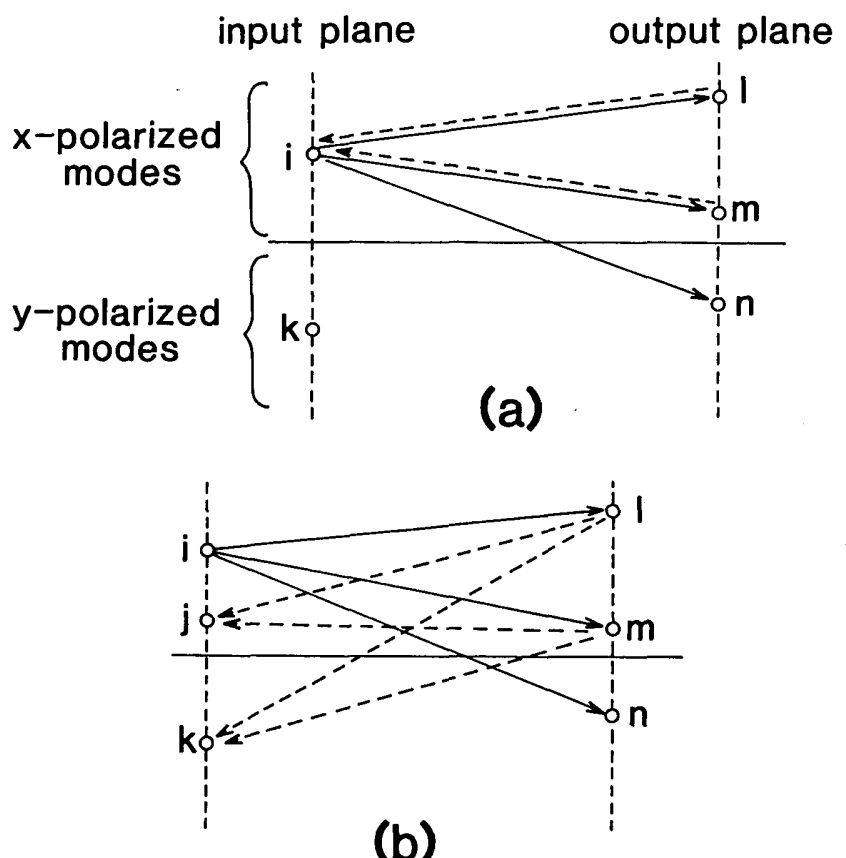

Fig. 2. Diagrammatic description of the formation of the field $\mathbf{E}^{(4)}$ : (a) deterministic phase-conjugate paths that result in true phase conjugation of the input field $\mathbf{E}^{(1)}$; (b) randomly scattered phaseconjugate paths that result in the noise. 
der is reflected by the PCM.] On the other hand, in Fig. 2(b) the remainder of the paths in the backward direction are random and different from those in the forward direction, and therefore because of the random interference at each mode at the input plane these field components form the noise $\mathrm{V}$ given in Eq. (24). We will see below that the total power of this noise is nearly the same as those of the true phase-conjugate field, but it is distributed essentially uniformly among all the fiber guided modes, independently of the input-beam N.A.'s. For this reason the noise power per mode is much smaller than that of the true phase-conjugate field, provided that the input field initially excites only a small fraction of the fiber guided modes (i.e., a small inputbeam N.A. is used) and that the detection is made within such a small input-beam N.A. In this case we can actually neglect such noise contributions designated by V in Eq. (24), and the field $\mathbf{E}^{(4)}$ can be the true phase-conjugate replica of the input field $\mathbf{E}^{(1)}$.

The correlations between the $2 N$ modes of the field $\mathbf{E}^{(4)}$ can also be expressed by means of the following $2 N \times 2 N$ Hermitian coherency matrix:

$$
\begin{aligned}
L^{(4)} & \equiv\left\langle\mathbf{E}^{(4)} \mathbf{E}^{(4)^{\dagger}}\right\rangle \\
& =|r|^{2} S L^{(1)^{*}} S^{\dagger} \\
& =|r|^{2}\left\{1 / 4 L^{(1)^{*}}+S_{2} L^{(1)^{*}} S_{2}^{\dagger}+1 / 2\left[S_{2} L^{(1)^{*}}+L^{(1)^{*}} S_{2}^{\dagger}\right]\right\} .
\end{aligned}
$$

In the right-hand side of the last equation above, the first term corresponds to a time-reversed polarization state of the input field $\mathbf{E}^{(1)}$, while the rest of the terms correspond to the noise. Since the expression given by Eq. (25) is linear in $L^{(1)}$, it is sufficient to consider the case of the $x$-polarized incidence for simplicity. In this case we can express the noise terms in Eq. (25), in terms of the submatrices $D$ and $Q$ given by Eqs. (23), as

$$
\begin{gathered}
S_{2} L^{(1)^{*} S_{2}^{\dagger}=}=\left[\begin{array}{cc}
D L_{x x}^{(1)^{*} D^{\dagger}} & D L_{x x}^{(1)^{*} Q} \\
Q^{\dagger} L_{x x}^{(1) *} D^{\dagger} & Q^{\dagger} L_{x x}^{(1)^{*}} Q
\end{array}\right], \\
\frac{1}{2}\left[S_{2} L^{(1)^{*}}+L^{(1)^{*}} S_{2}^{\dagger}\right]=\frac{1}{2}\left[\begin{array}{cc}
D L_{x x}^{(1)^{*}}+L_{x x}^{(1)^{*}} D^{\dagger} & L_{x x}^{(1)^{*} Q} \\
Q^{\dagger} L_{x x}^{(1)^{*}} & 0
\end{array}\right] .
\end{gathered}
$$

Each diagonal element in Eq. (26a) can be rewritten as

$$
\begin{aligned}
& {\left[D L_{x x}^{(1) *} D^{\dagger}\right]_{i i}=\left|D_{i l}\right|^{2}\left[L_{x x}^{(1)}\right]_{l l}+D_{i l} D_{i l^{\prime}}^{*}\left[L_{x x}^{(1)}\right]^{*} \underset{\left(l \neq l^{\prime}\right)}{l^{\prime},},} \\
& {\left[Q^{\dagger} L_{x x}^{(1)^{*}} Q\right]_{i i}=\left|Q_{l i}\right|^{2}\left[L_{x x}^{(1)}\right]_{l l}+Q_{l i}^{*} Q_{l^{\prime} i}\left[L_{x x}^{(1)}\right]^{*} \underset{\left(l \neq l^{\prime}\right)}{l^{\prime},},} \\
& {\left[D L_{x x}^{(1) *} Q\right]_{i i}=D_{i l} Q_{l^{\prime} i}\left[L_{x x}^{(1)}\right]_{l l^{\prime}}^{*},}
\end{aligned}
$$

where the summation over $l$ and $l^{\prime}$ is understood. In the above expressions Eq. (27a) corresponds to the noise power of the $x$-polarized $i$ th fiber guided mode of the field $\mathbf{E}^{(4)}$. This consists of the interference between the other initial modes that are finally coupled into the $x$-polarized $i$ th fiber guided mode through different scattering paths after the round-trip propagation. Likewise Eq. (27b) corresponds to the $y$-polarized noise power of the $i$ th fiber guided mode.

Each diagonal element in Eq. (26b) can also be rewritten

$$
\begin{aligned}
{\left[D L_{x x}^{(1)^{*}}+L_{x x}^{(1) * *} D^{\dagger}\right]_{i i} } & =2 \operatorname{Re}\left\{D_{i l}\left[L_{x x}^{(1)}\right]_{i i}^{*}\right\} \\
{\left[L_{x x}^{(1) *} Q\right]_{i i} } & =Q_{l i}\left[L_{x x}^{(1)}\right]_{i l}^{*},
\end{aligned}
$$

where the summation over $l$ is again understood. Equation (28a) corresponds to the interference between the true phase-conjugate field and the noise field at the $i$ th fiber guided mode of the $x$ polarization. This term is related to the residual polarization of the field $\mathbf{E}^{(2)}$. (Note that $D_{i l}=a_{i l}$ for $i \neq l$ and therefore the total power of this noise contribution, $\sum_{i=1}^{N}\left[D L_{x x}^{(1)^{*}}+L_{x x}^{(1)^{*}} D^{\dagger}\right]_{i i} / 2$, is equal to $q s_{0} / 2$; see Eqs. (16a), (20a), and (23a).\} We also note that this noise is distributed only inside the input-beam modal distribution $\left[L_{x x}^{(1)}\right]_{i i}$, i.e., this noise is $x$ polarized.

In order to estimate the ratio of this noise power to the true phase-conjugate beam power per mode, we consider the simple form of the scattering matrix elements, $M_{i j}=1 / \sqrt{2 N}$ $\exp i\left(\phi_{i j}\right) .^{10}$ Then, by inserting this form into Eqs. (27), we immediately see that $\left[D L_{x x}^{(1) *} D^{\dagger}\right]_{i i}$ and $\left[Q^{\dagger} L_{x x}^{(1)^{*}} Q\right]_{i i}$ are of the order of $s_{0} / N$ independently of the mode number $i$, where the total input power $s_{0}$ is given by Eq. (17a). It is therefore seen that the $x$ - and $y$-polarized noise powers given by Eqs. (27) do not differ from each other significantly at any $i$ th mode, so that the noise power of the field $\mathbf{E}^{(4)}$ is almost essentially uniformly distributed among all the fiber guided modes, independently of the input-beam N.A., i.e., of the distribution of $\left[L_{x x}^{(1)}\right]_{i i}$. We will see below that this noise is nearly completely depolarized. The ratio of the noise power to the true phase-conjugate beam power per mode is of the order of $M_{0} / N\left(M_{0}\right.$ is the number of the fiber guided modes that are excited initially). This ratio can be negligibly small when $M_{0} / N \ll 1$, justifying the fact that the noise $\mathbf{V}$ in Eq. (24) can be neglected for small N.A. inputs. In addition, the noise given by Eq. (28a) is of the order of $|q| s_{0} / M_{0}$, where $q$ is given by Eq. (20a). The ratio of this noise power to the true phase-conjugate beam power per mode is of the order of $|q|$, which is also much smaller than unity and is independent of the input-beam N.A.'s. Although this noise is $x$ polarized, its spatial structure is distorted because of the random phases. Therefore we refer to this as the polarized noise.

We are now in a position to evaluate quantitatively the polarization recovery of the input field $\mathbf{E}^{(1)}$ as a function of input-beam N.A.'s. Suppose that the whole power of the field $\mathbf{E}^{(4)}$ is detected. Then, according to the definition of the coherency matrix [see Eqs. (13)], the polarization state of the field $\mathbf{E}^{(4)}$ is expressed by means of the following $2 \times 2$ coherency matrix of the field $\mathbf{E}^{(4)}$ :

$$
\begin{aligned}
J^{(4)} & \equiv\left[\begin{array}{ll}
J_{x x}^{(4)} & J_{x y}^{(4)} \\
J_{x y}^{(4) *} & J_{y y}^{(4)}
\end{array}\right] \\
& =1 / 4|r|^{2} J^{(1)^{*}}+J_{\text {noise }}^{(4)}
\end{aligned}
$$

where

$$
J^{(1)}=\left[\begin{array}{cc}
s_{0} & 0 \\
0 & 0
\end{array}\right]
$$

and

$$
J_{\text {noise }}^{(4)}=\left\{\begin{array}{cc}
\operatorname{Tr}\left[L_{x x}^{(4)}\right]-1 /\left.4|r|\right|^{2} s_{0} & \operatorname{Tr}\left[L_{x y}^{(4)}\right] \\
\operatorname{Tr}\left[L_{x y}^{(4)}\right] & \operatorname{Tr}\left[L_{y y}^{(4)}\right]
\end{array}\right\} .
$$


After some calculations using Eqs. (25)-(27) together with the sum rules, each component of $J_{\text {noise }}^{(4)}$ can be written as

$$
\begin{aligned}
& {\left[J_{\text {noise }}^{(4)}\right]_{x x}=\left(\alpha_{1}+1 / 2 q s_{0}\right)|r|^{2},} \\
& {\left[J_{\text {noise }}^{(4)}\right]_{y y}=\left(1 / 4 s_{0}-\alpha_{1}\right)|r|^{2},} \\
& {\left[J_{\text {noise }}^{(4)}\right]_{x y}=\left(\alpha_{2}+1 / 4 v s_{0}\right)|r|^{2},}
\end{aligned}
$$

where

$$
\begin{aligned}
\alpha_{1} & =\operatorname{Tr}\left[D L_{x x}^{(1) *} D^{\dagger}\right], \\
\alpha_{2} & =\operatorname{Tr}\left[D L_{x x}^{(1) *} Q\right], \\
v & =\frac{2 \operatorname{Tr}\left[L_{x x}^{(1) *} Q\right]}{s_{0}},
\end{aligned}
$$

and the total noise power $P_{N}$ is found to be

$$
P_{N} \equiv \operatorname{Tr}\left[J_{\text {noise }}^{(4)}\right]=1 / 4|r|^{2} s_{0}(1+2 q) .
$$

It is seen from Eqs. (29)-(33) that almost one half of the reflected power [i.e., $|r|^{2}(1+2 q) s_{0} / 4$ ] is from the noise and the rest is from the true phase-conjugate beam. Since the polarization noise terms [given by $\alpha_{1} \mid r^{2}$ for the $x$ polarization and by $\left(s_{0} / 4-\alpha_{1}\right)|r|^{2}$ for the $y$ polarization] can be regarded as the probabilities that the initial $l$ th mode of the $x$ polarization is randomly coupled into all the fiber guided modes of the $x$ and $y$ polarizations after the round-trip propagation, these two terms may be almost equal, and then we have $\alpha_{1} \simeq$ $s_{0} / 8$. Consequently we can write the $2 \times 2$ coherency matrix of the noise as

$$
J_{\text {noise }}^{(4)} \simeq \lambda\left[\begin{array}{cc}
1+4 q & 2 v \\
2 v & 1
\end{array}\right],
$$

where $\lambda \equiv|r|^{2} s_{0} / 8$ and we have neglected $\alpha_{2}$, which is smaller than $v s_{0} / 4$ because of the complete phase mismatching. Note that, since $v$ is the same order of magnitude as that of $q$ and $u$, most of the noise expressed by relation (34) is nearly completely depolarized, except for the excess $x$-polarized noise denoted by $4 q \lambda$. The degree of polarization for the total integrated intensity of the field $\mathbf{E}^{(4)}$ is then given by

$$
P^{(4)} \simeq \frac{1+2 q}{2(1+q)}
$$

and the reflectivity $R$, defined as a ratio of the $x$-polarized reflected power to the input-beam power for the $x$-polarized input beam, is given by

$$
\begin{aligned}
R & \equiv \frac{J_{x x}^{(4)}}{s_{0}} \\
& =1 / 8|r|^{2}(3+4 q),
\end{aligned}
$$

where we neglect the second-order terms in $q$ and $v$. It is seen from expressions (35) that $P^{(4)}$ and $R$ depend on the residual polarization of the field $\mathbf{E}^{(2)}$, i.e., nonzero values of $q$. Furthermore, the degree of polarization recovery ${ }^{9} p$, which is defined as $p \equiv\left[J_{x x}^{(4)}-J_{y y}^{(4)}\right] /\left[J_{x x}^{(4)}+J_{y y}^{(4)}\right]$, i.e., the recovery of the linearly $x$-polarized component, is found to be equal to $P^{(4)}$ to first order in $q$ and $v$. If a linearly $y$ polarized light is used as an input, we obtain the same results as those given above with $q=2 D_{i l}^{\prime}\left[L_{y y}^{(1)}\right]_{i l}^{*} / s_{0}$. Finally, if the field $\mathbf{E}^{(2)}$ is completely depolarized (i.e., $q=v=0$ ), we obtain

$$
J_{\text {noise }}^{(4)} \simeq\left[\begin{array}{cc}
\lambda & 0 \\
0 & \lambda
\end{array}\right],
$$

so the noise field in the field $\mathbf{E}^{(4)}$ is completely depolarized and one half of the reflected power is equally distributed among all the fiber guided modes of both polarizations. In this case the degree of polarization $P^{(4)}$ of the total integrated intensity of the field $\mathbf{E}^{(4)}$ goes to 0.5 . This asymptotic behavior was also observed and explained theoretically by McMichael et al..$^{16}$

In practice, however, the input field $\mathbf{E}^{(1)}$ excites only a fraction of all the fiber guided modes (i.e., the input-beam N.A. is smaller than the fiber's N.A.). In addition, the detection is usually made only within the same (input-beam) N.A., and therefore the total noise power within the detection area is smaller than the total noise power discussed above. To see the effect of the input-beam N.A. on the degree of polarization $P^{(4)}$, we introduce the following modal partition functions for the true phase-conjugate field and the noise field, respectively:

$$
\begin{aligned}
\theta_{i} & \equiv \frac{\text { true phase-conjugate power in the } i \text { th mode }}{2 \lambda} \\
& =\frac{\left[L_{x x}^{(1)}\right]_{i i}}{s_{0}},
\end{aligned}
$$

$$
\begin{aligned}
\Lambda_{i} & \equiv \frac{\text { polarized noise power in the } i \text { th mode }}{4 q \lambda} \\
& =\frac{\operatorname{Re}\left[D L_{x x}^{(1) *}\right]_{i i}}{\left(\frac{q s_{0}}{2}\right)}
\end{aligned}
$$

$$
\begin{aligned}
\Delta_{i} & \equiv \frac{\text { depolarized noise power in the } i \text { th mode of each polarization }}{\lambda} \\
& =\frac{\left[D L_{x x}^{(1) *} D^{\dagger}\right]_{i i}}{\left(\frac{s_{0}}{8}\right)} \\
& \simeq \frac{\left[Q^{\dagger} L_{x x}^{(1) *} Q\right]_{i i}}{\left(\frac{s_{0}}{8}\right)} .
\end{aligned}
$$


Using these partition functions and the maximum mode number $M$ on the detection and then diagonalizing $J^{(4)}$, we can express the polarized power $P_{\text {pol. }}$ and the depolarized noise power $P_{M}$ as

$$
P_{\text {pol. }}=2 \lambda\left(\sum_{i=1}^{M} \theta_{i}+2 q \sum_{i=1}^{M} \Lambda_{i}\right)
$$

and

$$
P_{M}=2 \lambda \sum_{i=1}^{M} \Delta_{i}
$$

where we neglect the contribution of the off-diagonal elements in $L^{(4)}$ and also the second-order terms in $q$ and $v$. Then the degree of polarization $P^{(4)}$ and the reflectivity $R$ on the detection are given by

$$
\begin{aligned}
P^{(4)} & =\frac{P_{\text {pol. }}}{P_{\text {pol. }}+P_{M}} \\
& =\frac{1+2 q \beta_{1}}{1+2 q \beta_{1}+\beta_{2}}
\end{aligned}
$$

and

$$
R=\frac{1}{8}|r|^{2} \sum_{i=1}^{M} \theta_{i}\left(2+4 q \beta_{1}+\beta_{2}\right)
$$

where

$$
\beta_{1} \equiv \frac{\sum_{i=1}^{M} \Lambda_{i}}{\sum_{i=1}^{M} \theta_{i}}
$$

and

$$
\beta_{2} \equiv \frac{\sum_{i=1}^{M} \Delta_{i}}{\sum_{i=1}^{M} \theta_{i}},
$$

where expressions (34) and (37) were used to derive Eq. (39b). From Eq. (39a) we see that $P^{(4)}$ is again equal to the degree of polarization recovery $p$ and that $P^{(4)}$ approaches 0.5 as $M \rightarrow N$.

So far we have treated $r$ as a scalar value, i.e., $r$ is independent of the spatial structure of the field $\mathbf{E}_{x}^{(2)}$. Since the field $\mathbf{E}^{(2)}$ emitted from the fiber has a large field of view, the fidelity of the phase-conjugate field $\mathbf{E}^{(3)}$ reflected by the PCM may be degraded because of spatial frequency dependence of a phase-conjugate reflectivity. In what follows we consider the effect of this possible degradation on the polarization recovery.

We first decompose the field $\mathbf{E}^{(3)}$ into the true phaseconjugate field $\mathbf{E}_{x}^{(2)^{*}}$ multiplied by a scalar reflectivity $r_{0}$ and the possible wrong phase-conjugate field $\mathrm{E}_{w}^{(3)}$ :

$$
\begin{aligned}
\mathbf{E}^{(3)} & =r_{0} \mathbf{E}_{x}^{(2)^{*}}+\mathbf{E}_{w}^{(3)} \\
& =r_{0} \mathbf{E}_{x}^{(2)^{*}}+\left[r_{1}\right] \mathbf{E}_{x}^{(2)^{*}},
\end{aligned}
$$

where $\left[r_{1}\right]$ is a mode-dependent reflectivity that is a $2 N \times 2 N$ matrix. The explicit form of $\left[r_{1}\right]$ may depend on a type of the PCM. We then identify the scalar reflectivity $r$ used so far as an effective scalar reflectivity such that

$$
\begin{aligned}
\operatorname{Tr}\left[\mathbf{E}^{(3)} \mathbf{E}^{(3)^{\dagger}}\right]= & \left|r_{0}\right|^{2} \operatorname{Tr}\left[\mathbf{E}_{x}^{(2)^{*}} \mathbf{E}_{x}^{(2)^{* \dagger}}\right]+\operatorname{Tr}\left[\mathbf{E}_{w}^{(3)} \mathbf{E}_{w}^{(3)^{\dagger}}\right] \\
& +2 \operatorname{Re}\left\{\operatorname{Tr}\left[r_{0} \mathbf{E}_{x}^{(2)^{*}} \mathbf{E}_{w}^{(3)^{\dagger}}\right]\right\} \\
\equiv & |r|^{2} \operatorname{Tr}\left[\mathbf{E}_{x}^{(2)^{*}} \mathbf{E}_{x}^{(2)^{* \dagger}}\right]
\end{aligned}
$$

We then define the efficiency $\eta(0<\eta \leq 1)$ as

$$
\eta \equiv \frac{\left|r_{0}\right|^{2}}{|r|^{2}}
$$

which denotes a fractional power of the true phase-conjugate field $\mathbf{E}_{x}^{(2)}$ in the total power of the field $\mathbf{E}^{(3)}$ reflected by the PCM. For the output field $\mathbf{E}^{(4)}$ we can write [see Eq. (21) for comparison]

$$
\begin{aligned}
\mathbf{E}^{(4)} & =r_{0} S \mathbf{E}^{(1)^{*}}+M^{\prime} \mathbf{E}_{w}^{(3)} \\
& \equiv \mathbf{E}_{t}^{(4)}+\mathbf{E}_{w}^{(4)} .
\end{aligned}
$$

We further assume that each mode of the wrong phaseconjugate field $\mathbf{E}_{w}^{(4)}$ has no correlation to the other modes [i.e., the field $\mathbf{E}_{w}^{(4)}$ is completely depolarized], so that we can neglect the interference between $\mathbf{E}_{t}^{(4)}$ and $\mathbf{E}_{w}^{(4)}$ and the residual polarization of $\mathbf{E}_{w}^{(4)}$ (and therefore $2 \operatorname{Re}\left\{\operatorname{Tr}\left[r_{0} \mathbf{E}_{x}^{(2)^{* *}} \mathbf{E}_{w}^{(3) \dagger}\right]\right\}$ ). Under these assumptions we may express $J^{(4)}$ as

$$
\begin{aligned}
J^{(4)} & =J_{t}^{(4)}+J_{w}^{(4)} \\
& \simeq \eta \lambda\left[\begin{array}{cc}
2+4 q+1 & 2 v \\
2 v & 1
\end{array}\right]+2(1-\eta) \lambda\left[\begin{array}{cc}
1+q & 0 \\
0 & 1+q
\end{array}\right],
\end{aligned}
$$

where we have assumed the detection of the whole power of the field $\mathbf{E}^{(4)}$ and $\lambda$ is again given by $|r|{ }^{2} s_{0} / 8$. From relation (45) we see that the true phase-conjugate beam power is decreased by the factor $\eta$ but the depolarized noise power is increased because of the field $\mathbf{E}_{w}^{(4)}$. Consequently we can write the following general formulas for $P^{(4)}$ and $R$ [see Eqs. (39) for comparison]:

$$
P^{(4)}=\frac{1+2 q \beta_{1}}{1+2 q \beta_{1}+[1+2(\epsilon-1)(1+q)] \beta_{2}}
$$

and

$$
R=\frac{1}{8 \epsilon}|r|^{2} \sum_{i=1}^{M} \theta_{i}\left\{2+4 q \beta_{1}+[1+2(\epsilon-1)(1+q)] \beta_{2}\right\},
$$

where $\epsilon \equiv \eta^{-1}$ and we have assumed that the depolarized noise field $\mathbf{E}_{w}^{(4)}$ has the same partition function as $\Delta_{i}$.

We now consider the case of $M=M_{0}$ so that $\sum_{i=1}^{M} \theta_{i}=1$ and $\beta_{1}=1$, i.e., the detection aperture is the same as the input-beam N.A. According to the experimental observation $^{15}$ we let $\beta_{2}$ have a Gaussian distribution of the average depolarized noise intensity $I_{d}$ in each polarization in the detection plane (which is usually a far-field plane of the fiber end). Replacing a discrete modal intensity by a continuous one for a large $N$, that is,

$$
I_{d}=I_{0} \exp \left(-\frac{8 r^{2}}{\psi^{2}}\right)
$$




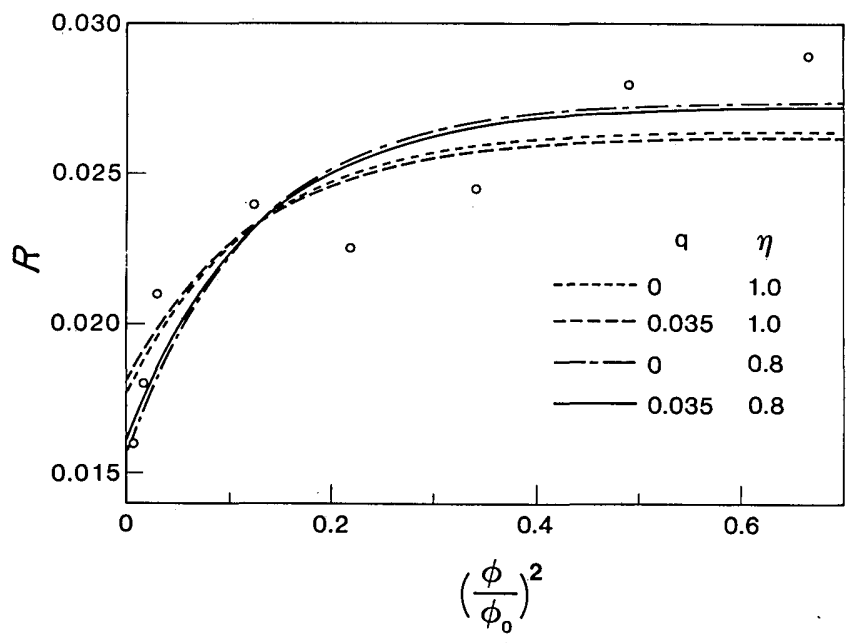

(a)

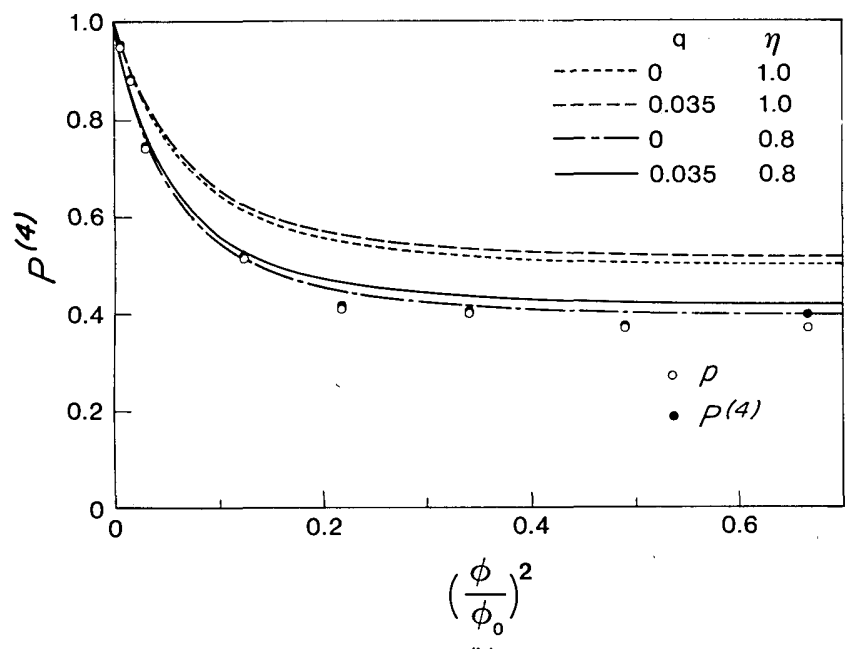

(b)

Fig. 3. Theoretical curves of (a) the phase-conjugate reflectivity $R$ and (b) the degree of polarization $P^{(4)}$ as a function of $\left(\phi / \phi_{0}\right)^{2}$ for $(q$, $\eta)=(0,1),(0.035,1),(0,0.8),(0.035,0.8)$. The Gaussian distribution $\left(\psi / \phi_{0}=0.5\right)$ of the depolarized noise intensity is assumed. The experimental data of (a) $R(0)$ and (b) $p(0)$ and $P^{(4)}(\bullet)$ are also shown.

where $\psi$ is an effective diameter of the depolarized noise intensity distribution in the detection plane, and $I_{0}=|r|^{2} s_{0} /$ $\psi^{2} \pi$ so that $\iint_{-\infty}^{\infty} I_{\mathrm{d}} \mathrm{d} x \mathrm{~d} y=|r|^{2} s_{0} / 8$, we then obtain

$$
\sum_{i=1}^{M} \Delta_{i} \simeq 1-\exp \left[-2\left(\phi / \phi_{0}\right)^{2} /\left(\psi / \phi_{0}\right)^{2}\right],
$$

where

$$
\phi_{0} \equiv \text { the input-beam diameter corresponding to } N
$$

and

$$
\phi \equiv \text { the input-beam diameter corresponding to } M,
$$

where the parameter $\left(\phi / \phi_{0}\right)^{2}$ is equal to $M / N$ and to (inputbeam N.A./fiber's N.A.) ${ }^{2}{ }^{23}$

Figure 3 shows the theoretical curves of $R$ and $P^{(4)}$ when $\psi /$ $\phi_{0}=0.5$ is used as a function of $\left(\phi / \phi_{0}\right)^{2}$. Four pairs of $(q, \eta)=$ $(0,1),(0.035,1),(0,0.8),(0.035,0.8)$ were used to see the effects of the residual polarization of the field $\mathbf{E}^{(2)}$ and the fidelity of the phase-conjugated field $\mathbf{E}^{(3)}$. [Note that a positive value of $q$ is consistent with the experiment in which the successive measurement of the Stokes parameters for the field $\mathbf{E}^{(2)}$ revealed that $s_{1}>0$. See Table 1.] The values of $q=0$ and $q=0.035$ used correspond to $P^{(2)}=0$ and $P=$ 0.05 , respectively, when $|q| \simeq|u|$ is assumed [see expression (19)]. The experimental data of $R$ and of $p$ and $P^{(4)}$ for the $x$-polarized input are also shown in Figs. 3(a) and 3(b), respectively. (In the experiment a self-pumped PCM using internal reflection ${ }^{19}$ was used. See Ref. 15 for detailed results.) Since the experimental data of $R$ included unwanted losses due to reflection and absorption by optical components, the proportional factors in the theoretical curves of $R$ were determined by the least-squares fits with the experimental data. In Fig. 3(a) it is seen that as $\left(\phi / \phi_{0}\right)^{2}$ (i.e., an input-beam N.A.) increases, $R$ also increases and finally saturates at a certain value. On the other hand, in Fig. 3(b) $P^{(4)}$ decreases steeply and finally saturates as $\left(\phi / \phi_{0}\right)^{2}$ increases. This occurs because the depolarized noise power within the detection aperture increases as $\left(\phi / \phi_{0}\right)^{2}$ increases, and finally it becomes comparable with the true phase-conjugate beam power when the input-beam N.A. is close to the fiber's N.A. We also see the increase of $P^{(4)}$ for $q=0.035$ since the polarized noise is added when $q$ is positive. We note that when the input-beam N.A. is much smaller than the fiber's N.A. [i.e., $\left(\phi / \phi_{0}\right)^{2} \ll 1$ ], then $P^{(4)}$ is close to unity independently of $q$ (i.e., almost complete polarization recovery is possible). This is so, as mentioned above, because the depolarized noise power is distributed among all the fiber guided modes so that for small input N.A.'s the depolarized noise power occupied within such a small fraction of all the fiber guided modes can be negligible compared with that of the true phase-conjugate beam. It is seen that the theoretical curves for $(q, \eta)=(0,0.8),(0.035,0.8)$ in Fig. 3, which are based on the assumption that the field $\mathbf{E}_{w}^{(4)}$ given in Eq. (44) is completely depolarized and distributed among all the fiber guided modes, are found to be in good agreement with the experimental data.

\section{SIGNAL-TO-NOISE RATIO OF THE RECONSTRUCTED SPATIAL INFORMATION}

In this section we consider the SNR of the reconstructed spatial information on phase conjugation. As was mentioned in Section 1, we employ the statistical treatment here. Goodman ${ }^{24}$ analyzed the SNR, which is defined as the ratio of the deterministic image intensity $I_{s}$ to the rms value $\sigma_{I}$ of the total image intensity at the same point, in a reconstructed image by a hologram. In our case it is necessary to derive the expression for the rms value of speckle noise intensity that results from the depolarized noise field reflected from the fiber on phase conjugation. In addition this speckle field, possessing both polarizations, is coherently added to the true phase-conjugate field, which acts as the $x$-polarized uniform coherent background signal.

The statistical properties of the sum of speckle patterns with coherent background intensities were studied theoretically by Ohtsubo et al..$^{25}$ for uncorrelated, partially developed speckles and by Steeger et al. ${ }^{26}$ for partially polarized, partially developed speckles. For the case of speckles from a multimode fiber, Steeger $e t ~ a l .{ }^{27}$ found experimentally that the partially polarized speckle field of a multimode fiber 
follows a negative exponential distribution in each linearly polarized speckle intensity and the speckle field is spatially stationary in its intensity and polarization statistics when all the fiber modes are equally excited. From these results we may calculate the SNR in our case (for simplicity we put $q=$ 0 and $\eta=1$ in the following calculation). Before the calculation, the following features of the field $\mathbf{E}^{(4)}$ should be repeated:

(1) The true phase-conjugate field acts as a coherent background intensity $I_{s}$ in the $x$-polarized intensity.

(2) The speckle noise field is completely depolarized so that there is no correlation between two orthogonal $x$ - and $y$ polarized components, and in an ensemble-averaged sense such speckle intensities in both polarizations are equal at one point in the detection plane.

(3) Each polarized component of the speckle noise field is fully developed, and therefore its intensity statistics obey a negative exponential distribution.

First consider the $x$-polarized intensity that is the sum of fully developed speckle intensity and coherent background intensity. The probability-density function of such intensity is well known and is called a modified Rician density. ${ }^{28}$ Its characteristic function is given by ${ }^{25}$

$$
\Phi_{I_{x}}(i v)=\frac{1}{\left(1-i v \bar{I}_{\text {noise }}\right)} \exp \left[-\frac{I_{s}}{\bar{I}_{\text {noise }}}+\frac{I_{s}}{\bar{I}_{\text {noise }}\left(1-i v \bar{I}_{\text {noise }}\right)}\right],
$$

where $\bar{I}_{\text {noise }}$ is the ensemble-averaged speckle noise intensity of one polarization at one point in the detection plane. For the $y$-polarized intensity, which is only fully developed speckle intensity, its characteristic function can be obtained from Eq. (49) with $I_{s}=0$ :

$$
\Phi_{I_{y}}(i v)=\frac{1}{\left(1-i v \bar{I}_{\text {noise }}\right)}
$$

Since there is no correlation between two orthogonal speckles, it follows immediately that the total characteristic function of interest is the product of Eqs. (49) and (50):

$$
\Phi_{I}(i v)=\frac{1}{\left(1-i v \bar{I}_{\text {noise }}\right)^{2}} \exp \left[-\frac{I_{s}}{\bar{I}_{\text {noise }}}+\frac{I_{s}}{\bar{I}_{\text {noise }}\left(1-i v \bar{I}_{\text {noise }}\right)}\right] .
$$

The rms noise intensity is then given by

$$
\begin{aligned}
\sigma_{I} & =\left[\bar{I}^{2}-(\bar{I})^{2}\right]^{1 / 2} \\
& =\left[\left.\frac{1}{(i)^{2}} \frac{\partial^{2}}{\partial v^{2}} \Phi_{I}(i v)\right|_{v=0}-\left(I_{s}+2 \bar{I}_{\text {noise }}\right)^{2}\right]^{1 / 2} \\
& =\left[2 \bar{I}_{\text {noise }}\left(\bar{I}_{\text {noise }}+I_{s}\right)\right]^{1 / 2} .
\end{aligned}
$$

It is seen from Eq. (52) that the rms noise intensity is expressed by $\bar{I}_{\text {noise }}$ and the interference term between the true phase-conjugate field and the depolarized noise field. The SNR can be written as

$$
\begin{aligned}
(\mathrm{SNR})_{x y} & \equiv \frac{I_{s}}{\sigma_{I}} \\
& =\gamma\left(\frac{2}{1+2 \gamma}\right)^{1 / 2},
\end{aligned}
$$

where $\gamma=I_{s} / 2 \bar{I}_{\text {noise }}$ is the beam-ratio parameter. ${ }^{28}$ If an analyzer (set to the $x$-polarization direction) is used to measure only the $x$-polarized component of the field $\mathbf{E}^{(4)}$, then the SNR can be given straightforwardly by

$$
(\mathrm{SNR})_{x}=\frac{2 \gamma}{(1+4 \gamma)^{1 / 2}} \text {. }
$$

To illustrate the dependence of these SNR's on the inputbeam N.A., we identify $I_{s}=|r|^{2} s_{0} /\left(\pi \phi^{2}\right)$ [i.e., the input is assumed to be a two-dimensionally uniform beam with the diameter $\phi$ so that the total power of the true phase-conjugate beam is $|r|{ }^{2} s_{0} / 4$ given in Eq. (29)] and $\bar{I}_{\text {noise }}=I_{d}$, where the average noise intensity $I_{d}$ is given by Eq. (47) in Section 4. Then the beam-ratio parameter $\gamma$ is given by

$$
\gamma=\frac{1}{2} \frac{\left(\frac{\psi}{\phi_{0}}\right)^{2}}{\left(\frac{\phi}{\phi_{0}}\right)^{2}} \text { at the center or the signal beam. }
$$

We therefore see the parametric dependence of the SNR on $P^{(4)}$ given by Eq. (39a) through $\left(\phi / \phi_{0}\right)^{2}$ (i.e., an input-beam N.A.).

Figure 4 shows the dependence of the two SNR's, as given by Eqs. (53) and (54), on $\left(\phi / \phi_{0}\right)^{2}$ at the center of the true phase-conjugate beam with the value of $\psi / \phi_{0}=0.5$ used in Fig. 3. The insets are photographs of the $x$-polarized phaseconjugate images of the letter $\mathrm{H}$ when $\left(\phi / \phi_{0}\right)^{2}=0.015$ [Fig. $4(\mathrm{a})]$ and $\left(\phi / \phi_{0}\right)^{2}=0.74$ [Fig. 4(b)]. It is seen that the two SNR's decrease rapidly when $\left(\phi / \phi_{0}\right)^{2}$ exceeds about 0.01 , i.e., the input-beam N.A. exceeds about $10 \%$ of the fiber's N.A. This is because, given a deterministically constant value of

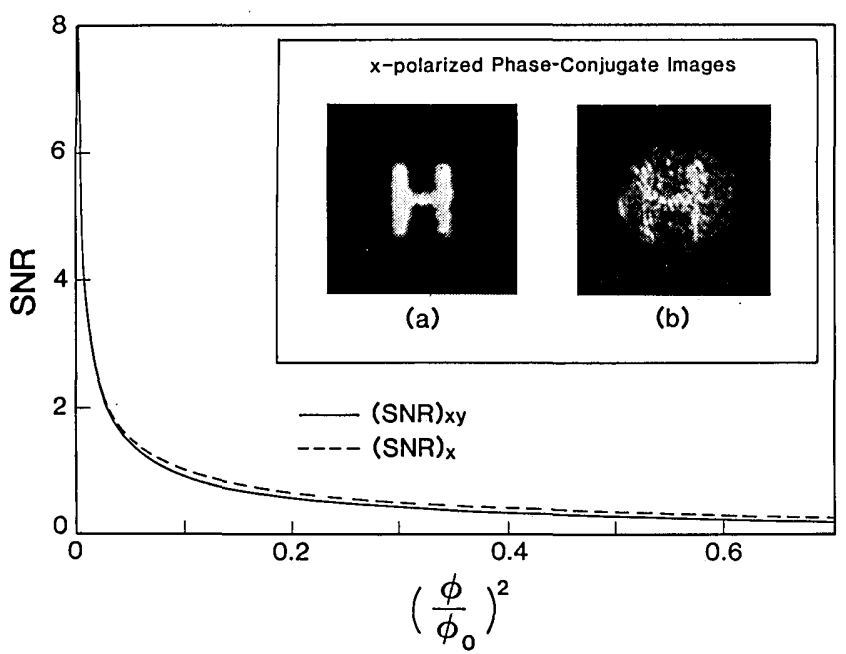

Fig. 4. Theoretical curves of the two SNR's, (SNR) $)_{x y}$ and (SNR) $)_{x}$, at the center of the signal beam as a function of $\left(\phi / \phi_{0}\right)^{2}$. The Gaussian distribution $\left(\psi / \phi_{0}=0.5\right)$ of the noise intensity is assumed. The insets are photographs of the $x$-polarized phase-conjugate images of the letter $\mathrm{H}$ for $(\mathrm{a})\left(\phi / \phi_{0}\right)^{2}=0.015$ and $(\mathrm{b})\left(\phi / \phi_{0}\right)^{2}=0.74$. 
the total power of the true phase-conjugate beam (i.e., $|r|^{2} s_{0} /$ 4), the intensity $I_{s}$ at the center of the phase-conjugate beam decreases as the input-beam N.A. increases, while the noise intensity $I_{\text {noise }}$ is almost constant independently of the input-beam N.A., resulting in a decrease of $\gamma$ (i.e., a decrease of the SNR). On the other hand, if the input-beam N.A. is much smaller than the fiber's N.A., then the intensity $I_{s}$ is much larger than the noise intensity $\bar{I}_{\text {noise }}$, resulting in a large value of $\gamma$ and therefore in the increase of the SNR. It is also seen that the two SNR's are almost the same over an entire range of the input-beam N.A.'s. This indicates that, although an analyzer is inserted in order to eliminate unwanted speckle noise of the orthogonal polarization, the improvement of the SNR is very small. ${ }^{29}$ The above-mentioned features are apparent from the two distinct photographs in Fig. 4. Finally it should be noted that the qualitative dependence of the SNR on the input-beam N.A. is the same as that of the degree of polarization shown in Fig. 3, although the SNR in a linear scale seems to be more sensitive to the input-beam N.A.

\section{CONCLUSIONS}

We have presented the detailed analysis of polarization and spatial information recovery in the FCPCM by means of a coherency matrix formalism. The basic physics behind this phenomenon can be explained in terms of the modal dispersal of information and the modal averaging by means of phase conjugation: because of the modal dispersal the initial information is distributed among all the fiber modes by which the original information can be recovered even when one field component of the mode-scrambled fields through the fiber is phase conjugated. In this case when the input information occupies only a small fraction of all the fiber guided modes, the (phase-mismatched) noise power per mode is much smaller (of the order of $N^{-1}$ ) than the true phaseconjugate power per mode because of the modal averaging and because these phase-mismatched fields are distributed equally among all the fiber guided modes. This gives nearly complete true phase conjugation of vector wave fronts for the input-beam N.A.'s that are much smaller than the fiber's N.A. On the other hand, since the total noise power is deterministically almost the same as the total power of the true phase-conjugate beam in the strong intermodal-coupling regime, serious degradation of the fidelity of true phase conjugation occurs when the input-beam N.A. is close to the fiber's N.A., and therefore the total noise power cannot be neglected. We have analyzed such effects theoretically, using the properties of the scattering matrix and the randomcoupling approximation. In addition, the effects of the modal-scrambling property of the fiber and the fidelity of phase conjugation by the PCM have been discussed. We have also analyzed the SNR in a reconstructed image by the FCPCM, based on the statistical properties of speckle noise under the assumption of the negative exponential probability-density function of speckle noise intensity distribution in both polarizations. It has been shown that the SNR is highly sensitive to the input-beam N.A. and seriously degrades when the input-beam N.A. exceeds about $10 \%$ of the fiber's N.A. This may be a criterion for the limit of the information-handling capacity when this simple FCPCM scheme is used for pictorial information processing ${ }^{12,30-38}$ and interferometry ${ }^{35,39}$ applications, but such a limit in spatial frequencies may not cause serious problems in signalprocessing applications, such as gyroscopes, ${ }^{40-43}$ sensors, ${ }^{11}$ and multichannel switching devices, ${ }^{13,14,44}$ when signal beams with large input-beam N.A.'s are not required for such applications. It should be noted finally that, although we have specifically considered the case of the $x$-polarized input, an input of any polarization state can also be considered straightforwardly by using the present formulation.

\section{APPENDIX A}

Express each element of the scattering matrix $M$ as

$$
M_{i j}=m_{i j} \exp \left(i \phi_{i j}\right)
$$

these elements are interrelated by the unitarity condition given by Eqs. (6) and (7). Suppose that there is strong intermodal coupling in the fiber so that the initially excited fiber modes at the input are redistributed among all the fiber modes during propagation. Then it is appropriate to assume that the amplitudes $m_{i j}$ are either nearly the same or symmetrically and widely distributed with respect to the diagonal elements $m_{i i}$, while the phases $\phi_{i j}$ are distributed essentially uniformly over the $-\pi-+\pi$ interval under the constraint of the unitarity condition. In this case the following random-coupling approximation may be adequate from Eqs. (6) and (7):

$$
\begin{aligned}
& \sum_{k=1}^{N}\left|\left(M_{i j}\right)_{k l}\right|^{2} \simeq \frac{1}{2}, \\
& \sum_{l=1}^{N}\left|\left(M_{i j}\right)_{k l}\right|^{2} \simeq \frac{1}{2},
\end{aligned}
$$

where $i, j=x, y$ and $k, l=1, \ldots, N$. In addition, because of the modal averaging over phase-mismatched terms, all the other cross terms are much smaller than unity.

\section{ACKNOWLEDGMENTS}

This research was supported by the U.S. Air Force Office of Scientific Research and by the U.S. Army Research Office, Durham, North Carolina.

\section{REFERENCES AND NOTES}

1. L. G. Cohen, "Measured attenuation and depolarization of light transmitted along glass fibers," Bell. Syst. Tech. J. 50, 23-42 (1971).

2. B. Ya. Zel'dovich and V. V. Shkunov, "Reversal of the wave front of light in the case of depolarized pumping," Sov. Phys. JETP 48, 214-219 (1978).

3. B. Ya. Zel'dovich and V. V. Shkunov, "Spatial-polarization wavefront reversal in four-photon interaction," Sov. J. Quantum Electron. 9, 379-381 (1979).

4. V. N. Blashchuk, B. Ya. Zel'dovich, A. V. Mamaev, N. T. Pilipetsky, and V. V. Shkunov, "Complete wavefront reversal of depolarized radiation under degenerate four-photon interaction conditions (theory and experiment)," Sov. J. Quantum Electron. 10, 356-358 (1980). 
5. G. Martin, L. K. Lam, and R. W. Hellwarth, "Generation of time-reversed replica of a nonuniformly polarized image-bearing optical beam," Opt. Lett. 5, 185-187 (1980).

6. S. Saikan and M. Kiguchi, "Generation of phase-conjugated vector wave fronts in atomic vapors," Opt. Lett. 7, 555-557 (1982).

7. P. Yeh, "Scalar phase conjugator for polarization correction," Opt. Commun. 51, 195-197 (1984).

8. I. McMichael, M. Khoshnevisan, and P. Yeh, "Polarizationpreserving phase conjugator," Opt. Lett. 11, 525-527 (1986).

9. K. Kyuma, A. Yariv, and S.-K. Kwong, "Polarization recovery in phase conjugation by modal dispersal," Appl. Phys. Lett. 49, 617-619 (1986).

10. A. Yariv, Y. Tomita, and K. Kyuma, "Theoretical model for modal dispersal of polarization information and its recovery by phase conjugation," Opt. Lett. 11, 809-811 (1986).

11. S.-K. Kwong, R. Yahalom, K. Kyuma, and A. Yariv, "Optical phase-conjugate correction for propagation distortion in nonreciprocal media," Opt. Lett. 12, 337-339 (1987).

12. Y. Tomita, K. Kyuma, R. Yahalom, and A. Yariv, "Demonstration of correction of amplitude distortion by modal dispersal and phase conjugation," Opt. Lett. 12, 1020-1022 (1987).

13. R. Yahalom, K. Kyuma, and A. Yariv, "Phase conjugation of mode scrambled optical beams: application to spatial recovery and interbeam temporal information exchange," Appl. Phys. Lett. 50, 792-794 (1987).

14. R. Yahalom, A. Agranat, and A. Yariv, "Optical threshold mechanism using fiber coupled phase conjugate mirror," in Digest of Topical Meeting on Photonic Switching (Optical Society of America, Washington, D.C., 1987), paper FB3.

15. Y. Tomita, R. Yahalom, and A. Yariv, "Fidelity of polarization and spatial information recovery using a fiber-coupled phase conjugate mirror," Opt. Lett. 12, 1017-1019 (1987).

16. I. McMichael, P. Yeh, and P. Beckwith, "Correction of polarization and modal scrambling in multimode fibers by phase conjugation," Opt. Lett. 12, 507-509 (1987).

17. K. Kim, L. Mandel, and E. Wolf, "Relationship between Jones and Mueller matrices for random media," J. Opt. Soc. Am. A 4, 433-437 (1987).

18. A. Yariv, Quantum Electronics, 2nd ed. (Wiley, New York, 1975), p. 357.

19. J. Feinberg, "Self-pumped, continuous-wave phase conjugator using internal reflection," Opt. Lett. 7, 486-488 (1982).

20. S. Solimento, B. Crosignani, and P. Di Porto, Guiding, Diffraction, and Confinement of Optical Radiation (Academic, Orlando, Fla., 1986), pp. 569-573.

21. A. W. Snyder and J. D. Love, Optical Waveguide Theory (Chapman and Hall, London, 1983), p. 292.

22. M. Born and E. Wolf, Principles of Optics, 5th ed. (Pergamon, New York, 1975), pp. 544-555.

23. Since the input-beam N.A. $\approx \phi / 2 f$ ( $f$ is a focal length of a lens) and $M / N_{\text {total }}=(\text { N.A./N.A.fiber })^{2} / 2(M$ is the number of modes corresponding to $\phi$ for one polarization; $N_{\text {total }}=2 N ; \mathrm{N}$.A.fiber is the fiber's N.A.), we obtain $M / N_{\text {total }}=\left(\phi / \phi_{0}\right)^{2} / 2$. In addition since $\phi^{2}$ is proportional to the solid angle $\Omega$ subtended by the beam launched into the fiber, then it also gives $\left(\phi / \phi_{0}\right)^{2}=\Omega / \Omega_{0}$.

24. J. W. Goodman, "Film-grain noise in wavefront-reconstruction imaging," J. Opt. Soc. Am. 57, 493-502 (1967).

25. J. Ohtsubo and T. Asakura, "Statistical properties of the sum of partially developed speckle patterns," Opt. Lett. 1, 98-100 (1977).

26. P. F. Steeger, T. Asakura, K. Zocha, and A. F. Fercher, "Statistics of the Stokes parameters in speckle fields," J. Opt. Soc. Am. A 1, 677-682 (1984).

27. P. F. Steeger, T. Asakura, and A. F. Fercher, "Polarization preservation in circular multimode optical fibers and its measurement by a speckle method," IEEE J. Lightwave Technol. LT-2, 435-441 (1984).

28. J. W. Goodman, "Statistical properties of laser speckle patterns," in Laser Speckle and Related Phenomena, 2nd ed., J. C. Dainty, ed. (Springer-Verlag, Berlin, 1984), pp. 9-75.

29. In practice, however, the use of the analyzer may improve the SNR better than that in the calculation because of the rejection of unwanted noise that is due to the backreflection from optical components.

30. A. Yariv, "Three-dimensional pictorial transmission in optical fibers," Appl. Phys. Lett. 28, 88-89 (1976).

31. A. Yariv, "On transmission and recovery of three-dimensional image information in optical fibers," J. Opt. Soc. Am. 66, 301306 (1976).

32. A. Gover, C. P. Lee, and A. Yariv, "Direct transmission of pictorial information in multimode optical fibers," J. Opt. Soc. Am. 66, 306-311 (1976).

33. V. V. Ivakhnik, V. M. Petnikova, M. S. Solomatin, and V. V. Shuvalov, "Compensation of wave front distortions in a thick inhomogeneous medium," Sov. J. Quantum Electron. 10, 373375 (1980).

34. G. J. Dunning and R. C. Lind, "Demonstration of image transmission through fibers by optical phase conjugation," Opt. Lett. 7, 558-560 (1982).

35. B. Fisher and S. Sternklar, "Image transmission and interferometry with multimode fibers using self-pumped phase conjugation," Appl. Phys. Lett. 46, 113-114 (1985).

36. B. Fisher and D. Peri, "Real-time three-dimensional imaging through fiber bundles by four-wave mixing," Opt. Lett. 10, 182183 (1985)

37. P. H. Beckwith, I. McMichael, and P. Yeh, "Image distortion in multimode fibers and restoration by polarization-preserving phase conjugation," Opt. Lett. 12, 510-512 (1987).

38. B. Fisher, S. Sternklar, and S. Weiss, "Photorefractive oscillation with intracavity image and multimode fiber," Appl. Phys. Lett. 48, 1567-1569 (1986)

39. S. Sternklar, S. Weiss, M. Segev, and B. Fisher, "Mach-Zehnder interferometer with multimode fibers using the double phaseconjugate mirror," Appl. Opt. 25, 4518-4520 (1986).

40. B. Fisher and S. Sternklar, "New optical gyroscope based on the ring passive phase conjugator," Appl. Phys. Lett. 47, 1-3 (1985).

41. P. Yeh, I. McMichael, and M. Khoshnevisan, "Phase-conjugate fiber-optic gyro," Appl. Opt. 25, 1029-1030 (1986).

42. I. McMichael and P. Yeh, "Self-pumped phase-conjugate fiberoptic gyro," Opt. Lett. 11, 686-688 (1986).

43. E. McMichael, P. Beckwith, and P. Yeh, "Phase-conjugate multimode fiber gyro," in Digest of Topical Meeting on Photorefractive Materials, Effects, and Devices (Optical Society of America, Washington, D.C., 1987), paper FA4.

44. H. J. Caulfield, J. Shamir, and Q. He, "Flexible two-way optical interconnections in layered computers," Appl. Opt. 26, 22912292 (1987). 